\title{
An accurate checking hole method used in hydraulic manifold CAD
}

\author{
Senior Engineser Song Jin-Qi \\ Fang Yong-Hong, Huang Yuan-Chao \\ Bei.jing Research Institute of Automation for \\ Machine Building Industry \\ No. 1 Jiaochangkou Dewai, 100011 Bei.jing, CHINA
}

\section{ARSTRACT}

An accurate checking hole method is presented in developed hydraulic manifold CAD. The method is to discretized the hole surface and get finite point set. then the point. set, are calculated by Boolean algebra. Besides, an optimum calculation process is developed to save the CPI working time while checking hole program is running.

\section{KEYWORDS}

Checking Hole Method, Manifold CAD

\section{INTRODUCTION}

There are some defects in checking holes program which is used in manifold design. For example, some program can only check holes which are parallel or perpendicular to the faces of manifold [1], others simplify cone as column or ball in the mathematical model of checking holes program 2$\}$. All this could not decide whether the holes connection satisfied our demanded design. These deferts would cause faults or failures in hydraul ic system design and manufacture.

In this paper, an accurate checking hole method is presented, the calculation method is as following.

First. HOL ${ }_{i}$ and HOL.i. which are demanded to check, are established in surface point. set HOIS $S_{1}\left(d_{1}, h_{1}, w\right)$ and $3 D$ point set HOL. $V_{\text {. }}$ $\left(d_{j}, h_{j}\right)$ separately. The $d, n$ and $w$ are diameter, length and wall of the hole separately. Second, HOL $S_{\mathrm{i}}\left(\mathrm{d}_{\mathrm{i}}, \mathrm{h}_{\mathrm{i}}, \mathrm{w}\right)$ is discretized to become finite point set \{point ( $x$, $y, z)\}$. The $(x, y, z)$ is the point value. Third, we decide whether the following formula is content

$$
\begin{aligned}
& \text { point }(x, y, z) \in H O L S_{1}\left(d_{r}, h_{1}, w\right) \quad \& \\
& \text { point }(x, y, z) \in H_{0 L V_{s}}\left(d_{s}, h_{s}\right)
\end{aligned}
$$

If content, HOL $\mathrm{L}_{i}$ and HOL $\mathrm{L}_{\mathrm{s}}$ are connected.

Using this method to check all holes in the manifold will spend longer running time. So an optimum calculation process is adopted. The process is to calculate shortest distance between two holes axis, and decide 
whether to used the accurate method. When the accurate method is adopted, it calculate just in a small dangerous area and give the minute wall between two holes.

\section{MATHEMATICAL EXPRESSIONS OF HOLES IN HYDRAULIC MANIFOLD}

Any two holes in manifold can be expressed by d, h, hc. (Fig. 1) Usually, The minimum wall between two holes is demanded. So, balls are made on the hole surface (Fig.2) we take one hole as HOLi and another as $\mathrm{HOL}_{\mathfrak{j}}$, make balls on HOL $\mathrm{i}$ surface. If the top of hole has cone, the mathematical model is:

$$
\begin{aligned}
& \mathrm{d}_{\mathrm{i}}{ }^{\prime}=\mathrm{d}_{\mathrm{i}}+2 \mathrm{w} \\
& \mathrm{hc}_{\mathrm{i}}{ }^{\prime}=0.5 \mathrm{~d}_{\mathrm{i}}{ }^{\prime} \tan 30^{\circ} \\
& \mathrm{h}_{\mathrm{i}}{ }^{\prime}=\mathrm{h}_{\mathrm{i}}+\mathrm{w} \tan 30^{\circ}
\end{aligned}
$$

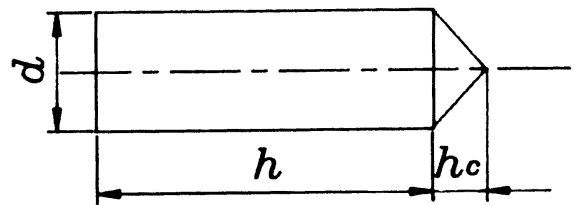

Fig. 1 Expression of hole

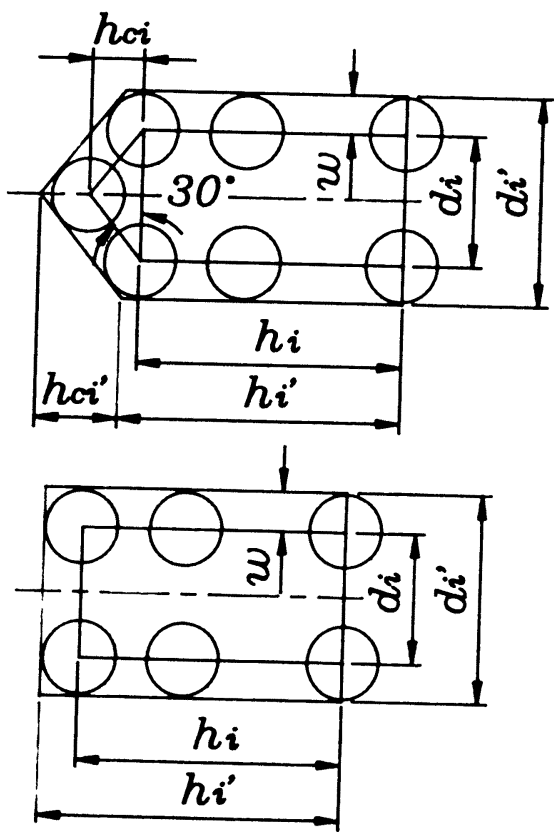

Fig. 2 make balls on the hole surface
If the hole is only a column, the model is

$$
\begin{aligned}
& d_{x^{\prime}}=d_{1}+2 w \\
& h_{1}^{\prime}=h_{1}+w
\end{aligned}
$$

\section{ESTABLISH FINITE POINT SET ON HOLE SURFACE}

Finite point set on HOL 1 surface will be set up in the coordinate system which HOLi is located (Fig. 3). The crossing point on column is:

$$
\begin{aligned}
& \mathrm{x}_{\mathrm{I}}=0.5 \mathrm{~d}_{\mathrm{i}}{ }^{\prime} \cos \theta \\
& \mathrm{y}_{\mathrm{i}}=0.5 \mathrm{~d}_{\mathrm{i}}{ }^{\prime} \sin \theta \\
& \mathrm{z}_{\mathrm{i}}=\mathrm{z}_{\mathrm{t}} \\
& \left(0<\theta<360^{\circ}\right) \\
& \left(0<\mathrm{z}_{\mathrm{t}}<\mathrm{h}_{\mathrm{i}}{ }^{\prime}\right)
\end{aligned}
$$

The crossing point on cone is:

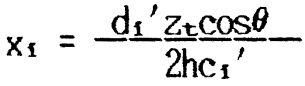

$$
\begin{aligned}
& y_{1}=\frac{d_{1}{ }^{\prime} z_{t} \sin \theta}{2 h_{c_{1}}{ }^{\prime}} \\
& z_{1}=h_{1}{ }^{\prime}+h c_{1}{ }^{\prime}-z_{t} \\
& \left(0<\theta<360^{\circ}\right) \\
& \left(0 \leqslant z_{t} \leqslant h_{c_{1}}\right)
\end{aligned}
$$

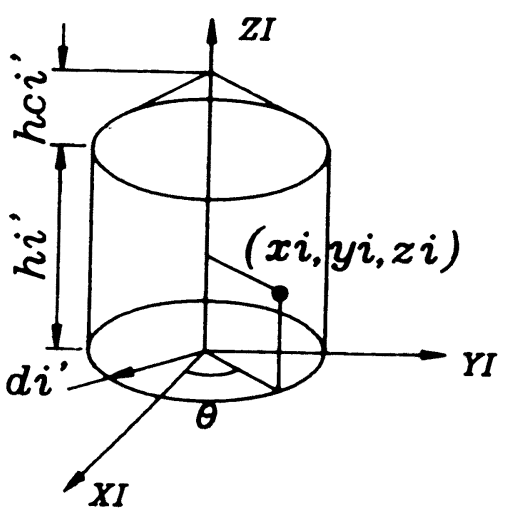

\section{Fig. 3 The description of crossing point}




\section{COORDINATE TRANSFORMATION}

Because the status values of HOL $\mathrm{L}_{1}\left(\mathrm{a}_{1}\right.$, $b_{1}, c_{1}, \alpha_{1}, \beta_{1}, \gamma_{1}$ ) and HOLs (as, $b_{5}, c_{3}$, $\left.\alpha_{s}, \beta_{s}, \gamma_{s}\right)$ are described in the world coordinate system (WCS), all point set in wich HOL I located (ICS) should be transformed to HOL, located (JCS) through the WCS in order to simplify checking hole mathematical model. The formula we used is as following:

$$
\begin{aligned}
& X=x_{-i n} \cos \alpha-y_{-1 n} \sin \alpha \\
& Y=y_{-i n} \cos \alpha-x_{-i n} \sin \alpha \\
& Y^{\prime}=Y \cos \beta-Z_{-i n} \sin \beta \\
& Z=Z_{-i n} \cos \beta+Y \sin \beta \\
& Z^{\prime}=Z_{\cos \gamma}-X_{\sin \gamma} \\
& X^{\prime}=X_{\cos \gamma}+Z \sin \gamma \\
& X_{\text {_out }}=X^{\prime}+a \\
& y_{\text {_out }}=Y^{\prime}+b \\
& Z_{\text {_out }}=Z^{\prime}+c
\end{aligned}
$$

in the formulas:

$x_{-i n}, y_{-i n}, z_{-i n}$ are the values in old coordinate.

$x_{\text {_out }}, \mathrm{y}_{\text {_out }}, \mathrm{z}_{\text {_out }}$ are the values in new coordinate.

$\alpha, \beta, \gamma$ are the angles rotated around $Z, X, Y$ axis seperately.

$a, b, c$ are the original point values of old coordinate in new one.

Replaced above parameters ( $x_{-i n}, y_{-i n}$, $\left.z_{- \text {in }}, \alpha, \beta, y, a, b, c\right)$ by point values in ICS and status values in WCS, and the point values in WCS can be obtained.

In WS, replaced by point values and status values multiply by -1 , the point values in the JCS can be obtained.

\section{CHECKING HOLE RULER}

In JCS, the following formulas are used to decide whether the two holes are connected or not.

The discriminants for the column hole are:

$$
X_{\text {_out }}{ }^{2}+Y_{\text {_out } 1}{ }^{2}<0.25 \mathrm{~d}^{2}
$$$$
0<z_{\text {_out }}<\mathrm{h}_{\mathrm{s}}
$$

The discriminants for hole with a cone are:

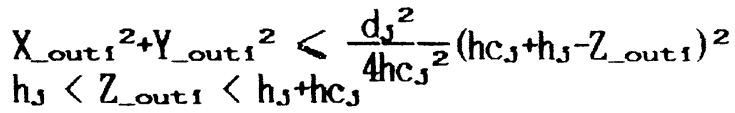

The discriminants for the holes which are in the same side and $d_{\mathfrak{i}}>d_{\mathfrak{j}}$ are:

$$
\begin{aligned}
& \alpha_{1}-\alpha_{J}=\beta_{1}-\beta_{J}=\gamma_{1}-\gamma_{J}=0 \\
& \left(a_{1}-a_{J}\right)^{2}+\left(b_{1}-b_{J}\right)^{2}+\left(c_{1}-c_{J}\right)^{2} \leqslant 0.25\left(d_{i}+d_{J}\right)^{2}
\end{aligned}
$$

If one of the above formulas is content and $w=0$, HOLi and HOLs is connected.

If none of the formulas is content and $W_{W} w_{d}$ is our demanded, HOLi and HOL 3 is not connect.

If $w=0$ and none of the formulas is content and $w<w_{d}$ and one of the three formulas is content, the wall is less than our demanded wall $\left(w_{d}\right)$, the two holes are dangerous. If we increase $w$ from 0 step by step, we could get the real minute wall.

\section{OPTIMUM PROCESS}

Usually, most of holes which connected or not is obviously. If all holes in manifold are calculated by accurate method, it will spend a long time. An optimum method is developed in the course of checking. The optimum method is as following.

First, dangerous holes can be selected by calculating the shortest distance (dis_i.j) between two center line segments ( $\overline{\mathrm{S}_{\mathrm{i}} \mathrm{e}_{\mathrm{i}}}$ \& $\overline{\mathrm{s}_{\mathrm{J}}}$ ) of two holes (Fig. 4). That is:

$$
\operatorname{dis}_{-1}=\operatorname{dis}\left(p_{k}, \overline{S_{j} e_{j}}\right)=\min \left(\operatorname{dis}\left(p_{1}, \overline{S_{j} e_{j}}\right)\right)
$$

in the formula:

$p_{1}(i=1,2, \ldots)$ is any point on segment line $\left(\overline{s_{i} e_{i}}\right)$.

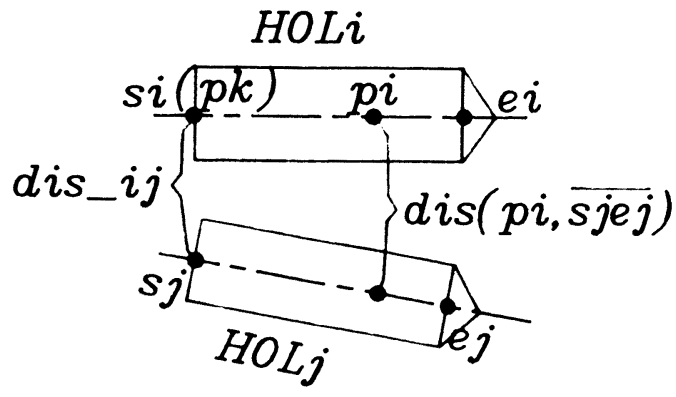

Fig. 4 The shortest distance between two line segments 
If dis is $>0.5\left(d_{i}+d_{5}\right)+w$, then HOL and HOLs is not connected and the demanded wall is content.

If dis $_{-1}<<\min \left(0.5 \mathrm{~d}_{\mathbf{i}}, 0.5 \mathrm{~d}_{\mathbf{j}}\right)$, then HOL and HOL 3 is connected.

Otherwise, the accurate checking hole method must be adopted to determine the holes connection.

When we search the $\min \left(\operatorname{dis}\left(\mathrm{p}_{\mathrm{f}}, \overline{\mathrm{S}_{s} \mathrm{e}_{\mathrm{J}}}\right)\right)$, the method of half segment, $\mathrm{si}_{\mathrm{i}} \mathrm{e}_{\mathrm{i}}$ divided internally is adopted to make $p_{i}$ converge to $p_{k}$ fast [3]. The value of $\operatorname{dis}\left(p_{1}, \overline{s_{5} e_{3}}\right)$ is calculated with analytic method shown in Fig. 5.

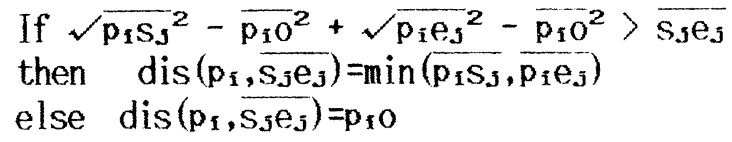

Second, after roughly selected and getting $p_{k}$, dangerous area between two holes can be decided and an accurate checking will be adopted only around the small area of $p_{k}$. It will decrease the working time as short as possible.

Besides, for the connected $\mathrm{HOl}_{i}$ and $\mathrm{HOL}_{3}$, the value of dis-1.j can be as a basis to determine whether the flow area is enough.

Figure 6 gives the simple diagram of procedure of the accurate checking hole method.

\section{EXAMPLE}

Fig. 7 is one cross-section of a manifold, table 1 is the part of information that the program outputed. It shows that hole one connect hole two; the minimum wall between hole one and hole three is $2.6 \mathrm{~mm}$, this is the dangerous wall if the demanded wall is $4 \mathrm{~mm}$; hole two connect hole four, but the shortest distance between two center line segments of two holes is just $7.2 \mathrm{~mm}$, so the flow area of these two holes is not enough.

Ilser can use these information to verify his drawing until get a satisfied result.

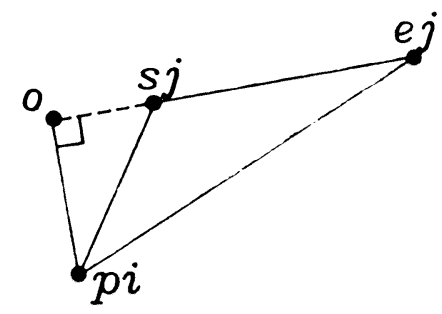

Fig. 5 The calculation of the $\operatorname{dis}(p i, \overline{s j e j})$

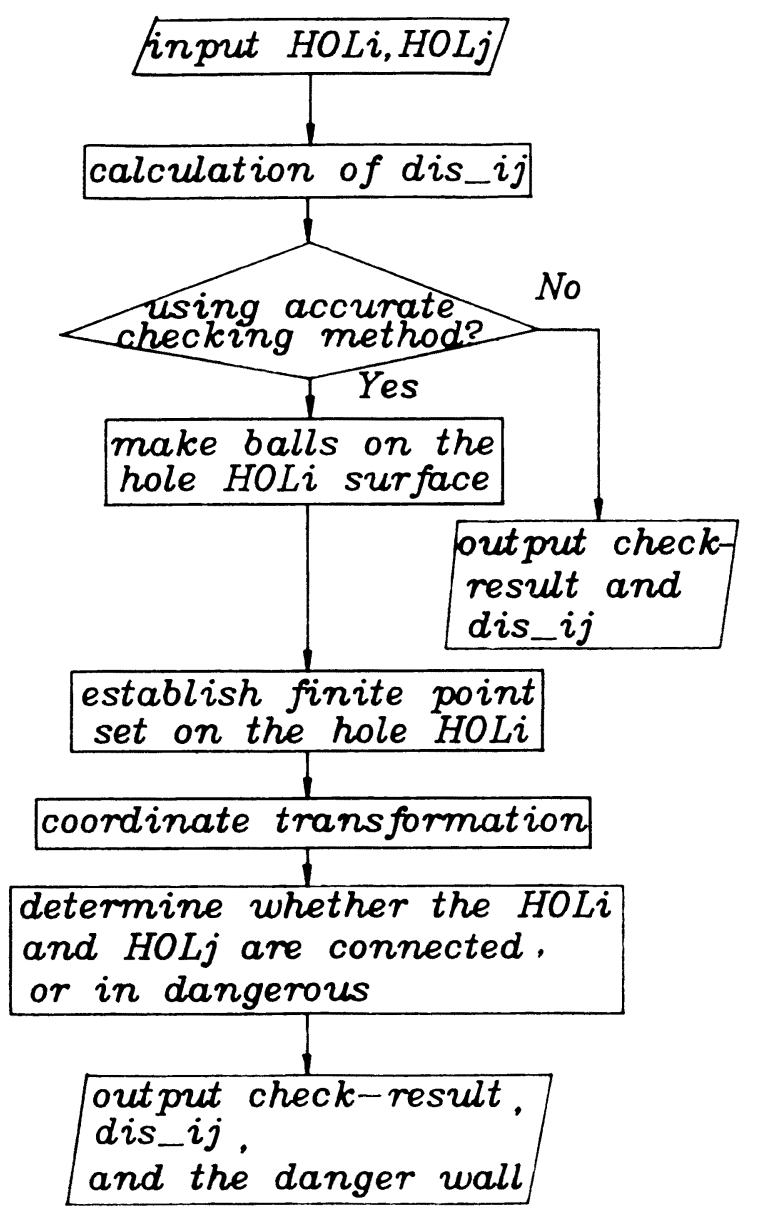

Fig. 6 Simplified flowchart of the program 


\section{CONCLUSION}

The calculating method has been applied to manifold design program, and has been widely used in many factories in China successfully. The users reflect that:

mastering is simple, operating is convenient, providing of information is comprehensive, the checking-result is procise.

TABLE 1

Information of the pragram outputed

\begin{tabular}{ccccr}
\hline i_No & j_No & Wall_dang & Hol_Cnt & Dist \\
\hline 1 & 2 & --- & Y & 0.5 \\
1 & 3 & 2.60 & N & 7.5 \\
1 & 4 & --- & N & 24.5 \\
2 & 3 & --- & N & 21.5 \\
2 & 4 & --- & Y & 7.2 \\
3 & 4 & --- & N & 35.8 \\
\hline
\end{tabular}

i_No, J_No--hole number;

Wall_dang--dangerous wall;

Hol_Cnt--connected holes;

Dist--shortest distance between

two holes's center line segments.

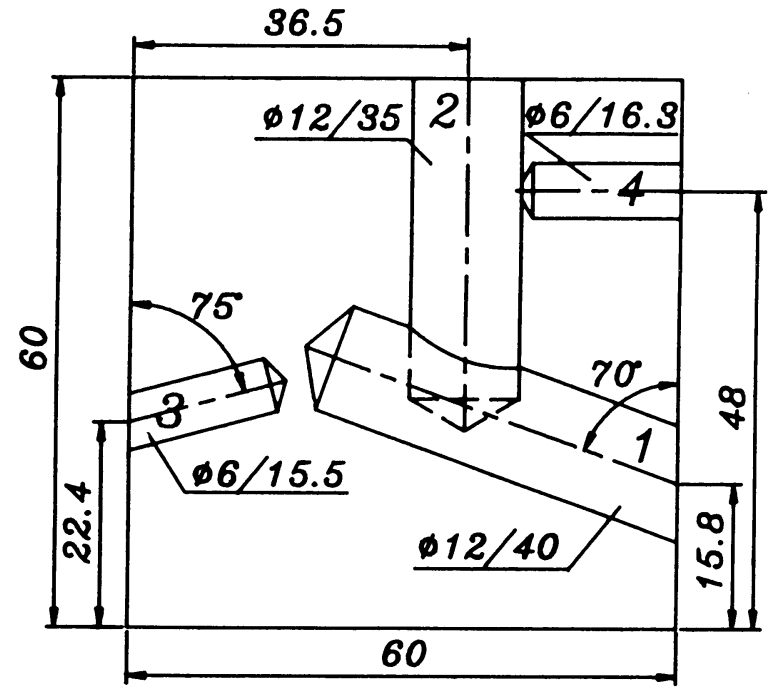

Fig. 7 One cross-section

of a manifold

\section{REFERENCES}

[1] Dalian University of Technology, Dal ian, China, Research Report on Assembly Hydraul ic System Design, Nov., 1990.

[2] Shanghai Jiao Tong University, Shanghai, China, Research Report on Manifold Design for Cartridge Valves, July, 1990.

[3] Beijing University and Qinhua University "Computational Method" Editorial Group, Computational Method, Science Press, Beijing, 1977. 